Bangladesh J Med Microbiol 2009; 03 (01): 1-3

Bangladesh Society of Medical Microbiologists

\title{
Editorial
}

\section{ESBL- A therapeutic challenge}

Extended spectrum beta-lactamase (ESBL) producing strains of Enterobacteriaceae have emerged as a major problem in hospitalised as well as community based patients. ${ }^{1,2}$ ESBLs are bacteria that produce ESBL enzymes that mediate resistance to extended-spectrum (third generation) cephalosporins (eg. ceftazidime, cefotaxime, and ceftriaxone) and monobactams (eg. aztreonam) but do not affect cephamycins (eg. cefoxitin and cefotetan) or carbapenems (eg. meropenem or imipenem).

ESBL infection is an opportunistic infection - it can be completely harmless in the healthy, but cause infection in the immunocompromised. These organisms are responsible for a variety of infections like urinary tract infection (UTI), septicaemia, hospital acquired pneumonia, wound infection, intra-abdominal abscess, brain abscess and device related infections. Although recent reviews have addressed the laboratory aspects in detection and classification of ESBLs they do not offer significant guidance about how to deal with these organisms in a particular clinical scenario. ${ }^{1,3}$ The present editorial aims at addressing the important clinical questions which are integral to the routine patient management.

This distinction can be made on the basis of some specific information like, a) Specimen type (isolates from physiologically sterile sites like blood, broncho alveolar lavage, tissue biopsy are to be taken seriously; whereas isolates from non-sterile sites like chronic wound swabs, sputum are more likely to be colonisers; isolates from catheterised specimen of urine are more likely to represent colonisation than isolates from mid stream urine; however, isolates from intravascular catheters/lines represent colonisation as well as potential sources of systemic infections), b) inflammatory parameters of the patient- white cell count, C-reactive protein, erythrocyte sedimentation rate, serum (infection is likely to be associated with derangement of these parameters), c) general condition of the patienttemperature, blood pressure, pulse rate, arterial oxygen

\section{Correspondence:}

Prof. KM Shahidul Islam

Head, Department of Microbiology

Begum Khaleda Zia Medical College

Sher-E-Bangla Nagar, Dhaka

E-mail : kmshahid2000@yahoo.com saturation, inotrope requirement, organ support requirement. These factors should be looked in combination rather than in isolation, and generally temporal trends in diagnostic parameters are more significant than a single value.

Non-antibiotic approach in the management of infections is a critical step in therapeutic decision making. Removal of the source of infection is crucial in management of most infections. ESBL is no exception. When the source of infection is a foreign body or a prosthetic device, removal or replacement of the device becomes all the more necessary. This is because infections associated with surgical implant and devices are associated with biofilm formation. Slow growth of microbes, together with restricted penetration of antibiotics inside biofilms makes eradication and treatment of device related infections difficult. The non-antibiotic approach in the management of ESBL related infections would include removal of a ESBL colonised intravascular line (central venous catheter, peripheral venous catheter), change of a colonised indwelling urinary catheter, drainage of an intra-abdominal or other intra-visceral abscesses, and removal of an infected prosthetic device- heart valve, prosthetic joint. It needs emphasis that in device related infections antibiotic therapy alone is unlikely to result in clinical improvement. ${ }^{4}$

The factors which determine the choice of antibiotics and other management options include: a) site of infection, b) severity of infection, c) presence of a prosthetic device or implant, d) metabolic parameters- liver and renal function, e) patient related factors such as age, pregnancy, lactation. Blood stream infections should be managed by carbapenems (e.g. imipenem, meropenem), whereas non-bacteraemic urinary tract infections especially lower urinary tract infections can be managed with a variety of antibiotics depending on its susceptibility. These include oral antibiotics like trimethoprim, nitrofurantoin, co-amoxiclav, mecillinam, or intravenous agents like aminoglycoside (gentamicin, amikacin), piperacillin-tazobactam, besides carbapenems (e.g., ertapenem). ${ }^{5}$

In vitro studies have demonstrated no synergy, additivity or antagonism in combination therapy (carbapenem + aminoglycoside). However, the bactericidal activity of imipenem in combination with amikacin was greater than that of imipenem alone. This was due to the faster killing rates of amikacin. ${ }^{6}$ It is in this background that in the treatment of life 
threatening infections like septicaemia, hospital acquired pneumonia, intra-visceral abscesses, carbapenems may be combined with a second agent (amikacin) for the first few days.

Meningitis (rare with ESBLs) and brain abscesses would require treatment with an agent which has good CSF penetration like meropenem (imi penem although efficacious, is better avoided in this setting as it is epileptogenic). The presence of an infected or colonised prosthesis complicates the management of ESBLs. Prosthesis removal should be the priority. However, if this were not done due to poor general condition of the patient, or physician's reluctance, long term combination therapy with a carbapenem and an aminoglycoside would be required.

Betalactam and betalactamase inhibitor combinations (coamoxiclav, piperacillin-tazobactam, etc.) are not the optimal therapy for serious infections due to ESBL-producing organisms. Although the inhibitors have significant activity against ESBLs in vitro, their clinical effectiveness against serious infections due to ESBL-producing organisms is controversial. The majority of ESBL producing organisms produce more than one betalactamase, often in different amount. Hyperproducing strains may produce enough betalactamase to overcome the effect of the inhibitor. Moreover, infections with high organism burden (intraabdominal collections, sepsis) may be associated with sufficient betal actamase production to overcome the effects of the betalactamase inhibitor. Finally, beta-lactams need to traverse outer membrane proteins through porin channels in order to reach the penicillin-binding proteins. Organisms such as K. pneumoniae may become deficient in these crucial outer membrane proteins, thereby rendering the betalactam betal actamase inhibitor combination clinically ineffective. However, they may be useful for less serious infections such as uncomplicated non-bacteraemic lower urinary tract infection because the infection is localised and the antibiotic is excreted in large amount through the urine ${ }^{7}$

The duration of therapy depends on the source of infection. In an uncomplicated non-bacteraemic urinary tract infection (UTI) 3 days of antibiotic therapy is considered sufficient ${ }^{8}$ Whereas, complicated UTI would necessitate 2 weeks of treatment Bacteraemia would require a minimum of 10-14 days of treatment apart from endocarditis and prosthetic joint infections, where 4-6 weeks of treatment is recommended. Tissue penetration of antibiotics is crucial in deciding therapy. ${ }^{9}$

For CNS infections aminoglycosides penetrate poorly through the blood brain barrier and should never be used in monotherapy. Similar logic applies to aminoglycosides in chest infections. Therapeutic antibiotic level monitoring is essential in aminoglycoside therapy. Pre doselevel should be
$<1 \mathrm{mg} / \mathrm{L}$ for gentamicin (for once daily dosing at $5 \mathrm{mg} / \mathrm{kg}$ ), and $<10 \mathrm{mg} / \mathrm{L}$ for amikacin.

Complain of "allergy" to various antibiotics are not uncommon. However, every effort must be made to distinguish true allergy, which is an IgE mediated type 1 hypersensitivity reaction from intolerance (which is nonimmunological and usually non-life threatening). In these situations therapy becomes difficult and probable choices in sensitive strains include aminoglycosides and quinolones (depending on antibiotic susceptibility). Injudicious and inappropriate usage may lead to selection of resistant organisms like MRSA, Stenotrophomonas maltophilia, metallo beta lactamase producing strains of Pseudomonas aeruginosa and Acinetabacter baumanii, and Candida spp. and development of antibiotic associated diarrhoea caused by Clostridiumdifficile.

It is clear from the above discussion that the treatment of ESBL producing organisms is a therapeutic challenge in view of the expense, use of broad-spectrum agents, frequent need of intravenous therapy, and infection control considerations. Management of systemically stable patients in hospital selting may give rise to cross infection, escal ated cost and increased morbidity. Use of parenteral antimicrobial agents, which can effectively be administered in an outpatient setting, can minimise a lot of these problems and improve patient compliance and quality of life. The availability of intravenous antibiotics like ertapenem and aminoglycosides (such as gentamicin) which can be administered once daily has given greater options in an OPAT setting. ${ }^{10}$

There was a time when virtually all ESBL isolates used to be reported from the hospital environment. However, with increasing use of broad spectrum antibiotics in the community setting and increasing number of ESBL positive patients who carry the organism from hospital to the community, more and more reports are emerging about community acquired outbreaks of ESBL infections. ${ }^{2}$ The information about the probable origin of the ESBL in a patient is important for infection control and epidemiologic reasons. The hospital microbiologist, infection control team, and clinician responsible for care need to be notified for adequate precautions and appropriate antibiotic therapy.

Infection control precautions like barrier nursing, cohorting of patients and nurses, contact precautions through the use of disposable gloves, gowns, and strict attention to hand washing are essential to limit its spread. Development of an infection control policy and hospital antibiotic prescribing guide should follow next. Education of medical and nursing staffs, patients, visitors and medical students through handouts, posters and meetings could play an important part. ESBL producers are intrinsically resistant to all cephalosporins and aztreonam (even if they exhibit invitro 
susceptibility). ${ }^{11}$ Interpretative comments can accompany microbiology reports to underline this fact. Co-resistance to quinolones and aminoglycosides are common. ${ }^{12}$ Quinolone antibiotics are strong selectors of ESBL producers and their use should be restricted as far as possible.

With ESBLs becoming an increasing problem in hospital and community setting, screening for the presence of these resistant pathogens (like MRSA screening) would ultimately become a necessity, especially in units with high antibiotic use. These would include high dependency units, postoperative wards, intensive care units, haematology, oncology, burn wards, orthopaedic and transplant centres. Screening for ESBL in large number of patients is a technical as well as financial challenge. A robust screening policy and an effective standard operating procedure would be crucial to minimise cost and confusion. Several specimens like rectal swabs, as well as urine, stool and sputum are tested in some centres to screen for resistant Gram negative bacilli (GNB). The choice and number of specimens in an institutional setting may ultimately depend on several factors like patient profile and resource availability. ${ }^{13}$

The management of ESBL requires a multi-disciplinary approach. Co-ordinated participation of microbiologists, clinicians, nursing personnel, hospital infection control team is essential. Therapeutic decision making requires a sound appreciation of clinical perspective Potential for screening exists but it must be tailored to the institutional need and patient profile. The petri-dish has long inspired our admiration for ESBL producers. It is time to extend our appreciation to the patients who are the ul timate sufferers.

\section{Prof. K M Shahidul Islam \\ Head, Department of Microbiology \\ BegumK Khaleda Zia Medical College \\ Sher-E-Bangla Nagar, Dhaka}

\section{R eference:}

1. Chaudhury U, Aggarwal R. Extended spectrum beta-lactamases (ESBL)- An emerging threat to clinical therapeutics. Indian J Med Microbiol 2004;22:75-80.

2. Rodriguez-Bano J , Navarro MD, Romero L, MartinezMartinez L, Muniain MA, Perea EJ , et al. Epidemiology and clinical features of infections caused by extended-spectrum beta- lactamase-producing Escherichia coli in nonhospitalized patients. J Clin Microbiol 2004;42:1089-94.

3. Bradford PA. Extended-Spectrum-Lactamases in the 21st Century: Characterization, epidemiology, and detection of this important resistance threat. Clin Microbiol Rev 2001;14:933-51.

4. DarouicheRO. Treatment of infections associated with surgical implants. N Engl J Med 2004;350:1422-9.

5. Iannini PB. Hospital-acquired Urinary Tract I nfections: Implications for Treatment and Prevention. Curr Tr Op I nfect Dis 2002;4:15-20.

6. Pattharachayakul S, Neuhauser MM, Quinn JP, Pendland SL. Extended-spectrum beta-lactamase (ESBL)-producing Klebsiella pneumoniae:activity of single versus combination agents. J Antimicrob Chemother 2003;51:737-9.

7. Paterson DL. B-Lactamase inhi bitors and new beta -lactamases. Curr Treat Opt I nfect Dis 1999;1:85-87.

8. Lutters M, Vogt N. Antibiotic duration for treating uncompl icated, symptomatic lower urinary tract infections in el derly women (Cochrane Review). In: The Cochrane Library, Issue 3. Chichester, UK: J ohn Wiley \& Sons, Ltd; 2004. http:// www.cochrane.org/cochrane/revabstr/ab001535.htm

9. Bloomfield P, Hodson EM, Craig JC. Antibiotics for acute pyel onephritis in children (Cochrane Review). In:The Cochrane Library, Issue 3. Chichester, UK:J ohn Wiley \& Sons, Ltd; 2004. http://www.cochrane.org/cochrane/revabstr/ ab003772.htm

10. TiceAD. Ertapenem: a new opportunity for outpatient parenteral antimicrobial therapy. J Antimicrob Chemother 2004;53:83-6.

11. Paterson DL, Ko WC, Von Gottberg A, Casellas JM, Mulazimoglu L, Klugman KP, et al. Outcome of Cephal osporin Treatment for Serious I nfections Due to A pparently Susceptible Organi sms Producing Extended-Spectrum - Lactamases: Implications for the Clinical Microbiology Laboratory. J Clin Microbiol 2001;39:2206-12.

12. Mathai D, Rhomberg PR, Biedenbach DJ , J ones RN. India Antimicrobial Resistance Study Group. Evaluation of the in vitro activity of six broad-spectrum beta-lactam antimicrobial agents tested against recent clinical isolates from India: a survey of ten medical center laboratories. Diagn Microbiol Infect Dis 2002;44:367-77.

13. Fergusson J. On behalf of theAICA National Advisory Board. Aust I nfect Con 2002;7; http://www.aica.org.au/docs/about/ downloads/definition2.pdf 